\title{
Исследование пространственной и токовой динамики оптических потерь в полупроводниковых лазерных гетероструктурах методом оптического зондирования
}

\author{
() П.С. Гаврина, О.С. Соболева, А.А. Подоскин, А.Е. Казакова, В.А. Капитонов, С.О. Слипченко, Н.А. Пихтин \\ Физико-технический институт им. А.Ф. Иоффе Российской академии наук, \\ 194021 Санкт-Петербург, Россия \\ E-mail: gavrina@mail.ioffe.ru
}

Поступила в Редакцию 30 марта 2020 г.

В окончательной редакции 7 апреля 2020 г.

Принята к публикации 7 апреля 2020 г.

Проведены исследования пространственно-временно́й динамики оптических потерь и концентрации носителей заряда в гетероструктуре полупроводникового лазера с сегментированным контактом с использованием методики накачки-зондирования (pump-probe), основанной на вводе зондирующего излучения с длиной волны 1560 нм в исследуемый кристалл полупроводникового лазера на основе гетероструктуры $\mathrm{AlGaAs} / \mathrm{InGaAs} / \mathrm{GaAs}$, излучающего на длине волны 1010 нм. Показано, что использование зондирующего излучения на длине волны 1560 нм позволяет обеспечить чувствительность измерения внутренних оптических потерь не хуже $1 \mathrm{~cm}^{-1}$. Использование сегментированной конструкции области токовой накачки позволило оценить абсолютную величину внутренних оптических потерь. Показано, что изменение конфигурации собственных мод лазера Фабри-Перо влияет на распределение носителей заряда и внутренних оптических потерь как в области токовой накачки, так и в пассивной, не прокачиваемой током, части лазерного кристалла.

Ключевые слова: полупроводниковый лазер, показатель поглощения, сегментированный контакт, оптическое зондирование.

DOI: $10.21883 /$ FTP.2020.08.49644.9400

\section{1. Введение}

Для большинства полупроводниковых приборов информация о перераспределении концентраций свободных носителей заряда в структуре является принципиально важной, так как позволяет определить условия и особенности работы прибора, связать выходные характеристики с параметрами структуры и режимом возбуждения. К таким приборам можно отнести биполярные транзисторы и тиристоры, различные типы диодов и т.д. С одной стороны, фундаментальные механизмы, определяющие базовые принципы работы таких приборов, достаточно хорошо изучены $[1,2]$ и в большинстве случаев существуют хорошо проработанные многомерные модели [3]. Однако применимость таких подходов для разработки новых типов приборов с требуемыми характеристиками ограничена низкими уровнями возбуждения, что затрудняет разработку приборных структур для работы в условиях пропускания высоких плотностей тока (десятки кА $/ \mathrm{cm}^{2}$ ) или включения процессов, приводящих к существенным пространственным неоднородностям распределения носителей заряда, электрического поля, излучательной рекомбинации и т.д. При этом информация о распределении носителей заряда в том или другом рабочем режиме доступна только из разработанных расчетных моделей, что не позволяет получить точные данные, поскольку все модели ограничены используемыми допущениями. Задачей настоящей работы является развитие подхода для прямого экспериментального измерения пространственного распределения избыточных носителей заряда в слоях различных типов гетероструктур. Одним из объектов, требующих подробных измерений, являются мощные полупроводниковые лазеры. Современные полупроводниковые лазеры способны работать при плотностях токов накачки в десятки кА $/ \mathrm{cm}^{2}$, что приводит как к перераспределению носителей в слоях гетероструктуры по сравнению с околопороговым режимом работы, так и к изменению оптических потерь на свободных носителях заряда [4,5]. В рамках данной работы исследованы особенности методики оптического зондирования, основанной на анализе оптических потерь сканирующего лазерного излучения для исследования пространственного распределения оптических потерь и носителей заряда в слоях полупроводниковой диодной гетероструктуры. Данная работа является продолжением цикла исследований, посвященных разработке методики измерения зависимости оптических потерь в мощных полупроводниковых лазерах от тока накачки [6,7]. Особенностью ранее представленных работ является то, что используемые режимы работы и конструкции обеспечивали однородное распределение носителей в активном кристалле. В рамках предлагаемой работы сделана первая попытка измерений для структур и условий накачки, обеспечивающих неоднородное в пространстве распределение носителей заряда. Возможности оценки пространственного распределения являются важным преимуществом, так как позволят в будущем проводит исследования распределения носителей в структуpax с ярко выраженной пространственной локализаци- 

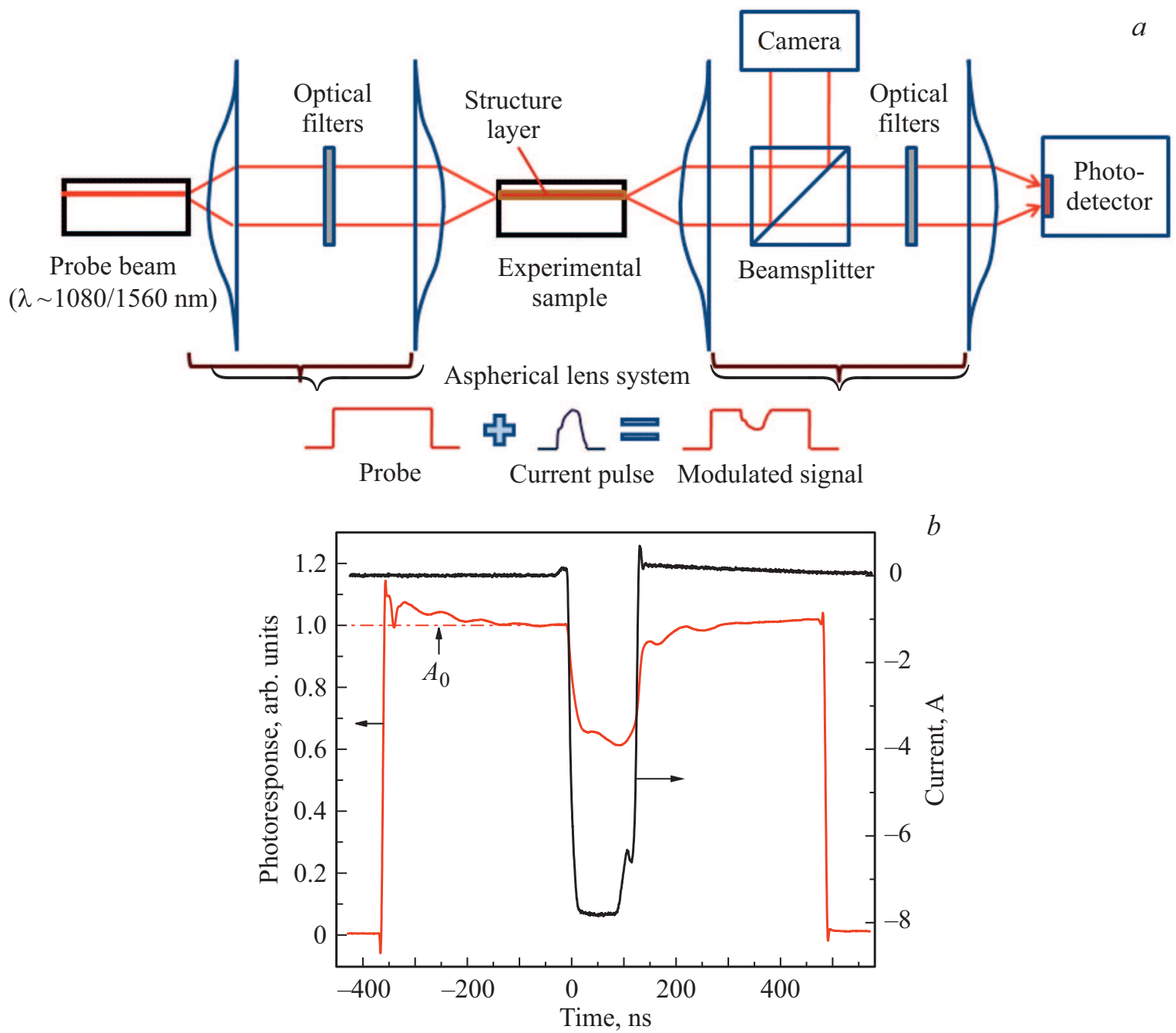

Рис. 1. Оптическая схема эксперимента $(a)$; общий вид промодулированного поглощением на свободных носителях импульса зондируюшего излучения и измеряемого импульса тока $(b)$.

ей, например, лазерах-тиристорах или фототранзистоpax [8-10], что необходимо для разработки и оптимизации параметров приборов на основе подобных структур.

2.

\section{1. Методика и экспериментальная схема измерений}

В основе методики, используемой в этой работе, лежит на первый взгляд простая идея: поглощение излучения на свободных носителях будет тем сильнее, чем больше концентрация носителей заряда (например, см. [11]). Для большинства полупроводниковых гетероструктур протекание электрического тока сопровождается накоплением избыточных носителей заряда в ее слоях. В результате протекающий через образец электрический ток приведет к снижению интенсивности (модуляции интенсивности) введенного в образец зондирующего излучения от внешнего источника за счет поглощения на свободных носителях заряда. Оценивая амплитуду модуляции, мы можем сделать выводы об изменении показателя поглощения излучения на свободных носителях заряда. Кроме того, имея возможность перемещать относительно друг друга источник зондирующего излучения и исследуемый образец, можно обеспечить необходимое пространственное разрешение. Временно́е разрешение будет зависеть от используемой измерительной аппаратуры, в нашем случае оно составляло 1 нс. Подобная методика применялась в [6,7] для оценки средних внутренних оптических потерь в мощных полупроводниковых лазерах, а также для выявления положения и размеров токовых шнуров в полупроводниковых лазерах-тиристорах полосковой конструкции [12].

Традиционным соотношением, которое позволяет определить коэффициент поглощения излучения при прохождении через исследуемую среду, является закон Бугера-Ламберта-Бера [2]:

$$
I^{\prime}=I_{0} \exp (-\alpha L)
$$




$$
\alpha=-1 / L \ln \left(\frac{I^{\prime}}{I_{0}}\right),
$$

где $I^{\prime}-$ интенсивность излучения на выходе из исследуемой среды, $I_{0}-$ интенсивность излучения на входе в исследуемую среду, $\alpha-$ коэффициент поглощения в среде, $L-$ длина прохождения излучения в исследуемой среде. Методики, описанные в работах [6,7], позволяют оценить лишь изменение показателя поглощения. Это связано с тем, что точная оценка коэффициента ввода излучения в волновод гетероструктуры затруднительна, и в этом случае величина интенсивности излучения на входе в исследуемый образец $I_{0}$ остается неизвестной.

Решением проблемы может стать использование для калибровки методики таких экспериментальных образцов, в которых длина прохождения излучения в поглощающей среде $L$ может контролируемо изменяться. Тогда (1) для длин $L_{1}, L_{2}\left(L_{1} \neq L_{2}\right)$ даст для интенсивностей соответственно

$$
\begin{aligned}
& I_{1}=I_{0} \exp \left(-\alpha L_{1}\right), \\
& I_{2}=I_{0} \exp \left(-\alpha L_{2}\right) .
\end{aligned}
$$

Коэффициент поглощения может быть определен из отношения $I_{1}$ и $I_{2}$ и разности длин $L_{1}$ и $L_{2}$ :

$$
\alpha=\frac{1}{L_{2}-L_{1}} \ln \left(\frac{I_{1}}{I_{2}}\right) .
$$

Выражение (4) применимо исключительно в случае постоянного коэффициента ввода излучения в образец, при этом сама величина интенсивности $I_{0}$ уже не будет иметь принципиального значения для случая малосигнального приближения.

Для ввода излучения в определенную область образца была использована оптическая схема, показанная на рис. 1,a. Схема состояла из системы асферических линз, отвечающих за коллимацию излучения от лазера засветки, ввод излучения в интересующий слой гетероструктуры, вывод излучения из кристалла на фотодетектор и визуализирующую камеру (через деление пучка на поляризационно-независимом делительном кубе), а также оптических фильтров, позволяющих пропускать зондирующее излучение и отсекать собственное излучение образца.

Засветка осуществлялась оптическими импульсами длительностью $\sim 1$ мкс, исследуемый образец прокачивался токовым импульсом длительностью $\sim 100$ нс. Общий вид промодулированного поглощением на свободных носителях импульса зондирующего излучения (слева) и измеряемого импульса тока (справа) можно видеть на рис. $1, b$. Через $A_{0}$ обозначена амплитуда фотоотклика до начала модуляции, в нашем эксперименте при нормировке она была принята равной единице.

\section{2. Экспериментальные образцы}

Чтобы оценивать именно поглощение на свободных носителях, зондирующее излучение должно быть более длинноволновым по отношению к спектру усиления исследуемого образца. В противном случае мы будем наблюдать либо усиление исходного сигнала, либо интенсивное межзонное поглощение. В нашей работе в качестве источника зондирующего излучения применялся полупроводниковый лазер полосковой конструкции с выходной апертурой 100 мкм, излучающий на длине волны 1560 нм (спектр 2 на рис. 2,a).

Образцы представляли собой гетероструктуру полупроводникового лазера, состав которой приведен в таблице, спектр излучения лазера представлен на рис. 2, $a$ (спектр 1).

В процессе проведения постростовых операций к $p$-стороне гетероструктуры был изготовлен полосковый контакт сегментированной конструкции. Ряды рабочих сегментов разделялись пассивными секциями, имеющими сплошной контакт. Вид сверху на общую конструкцию образов представлен на рис. $2, b$, где $1,2, \ldots \mathrm{N}$ положения рабочих сегментов. Длина образцов составляла $\sim 2800$ мкм. Разделительные мезаканавки между
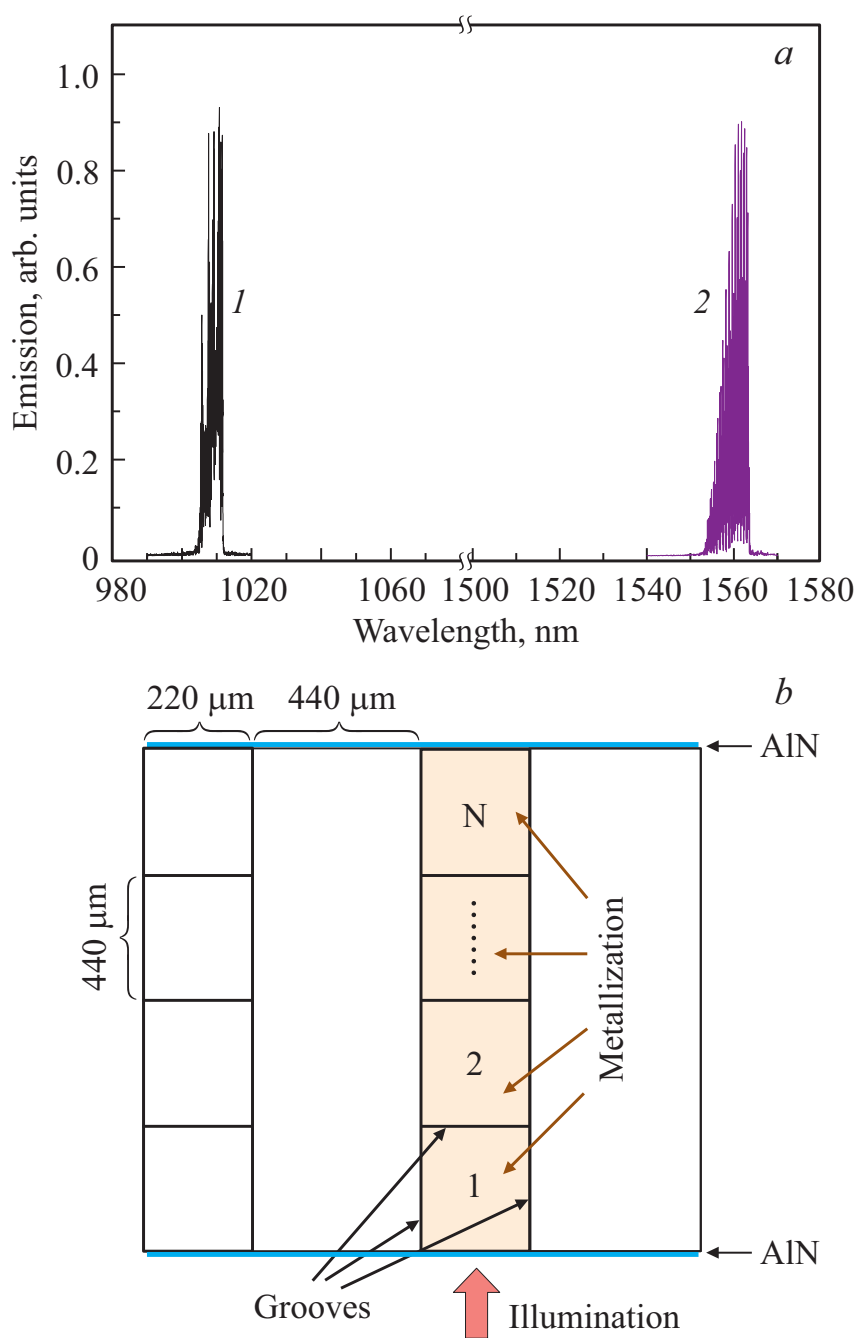

Рис. 2. $a-$ спектры излучения исследуемых образцов (1) и лазера засветки (2); $b$ - экспериментальные образцы, вид сверху. 

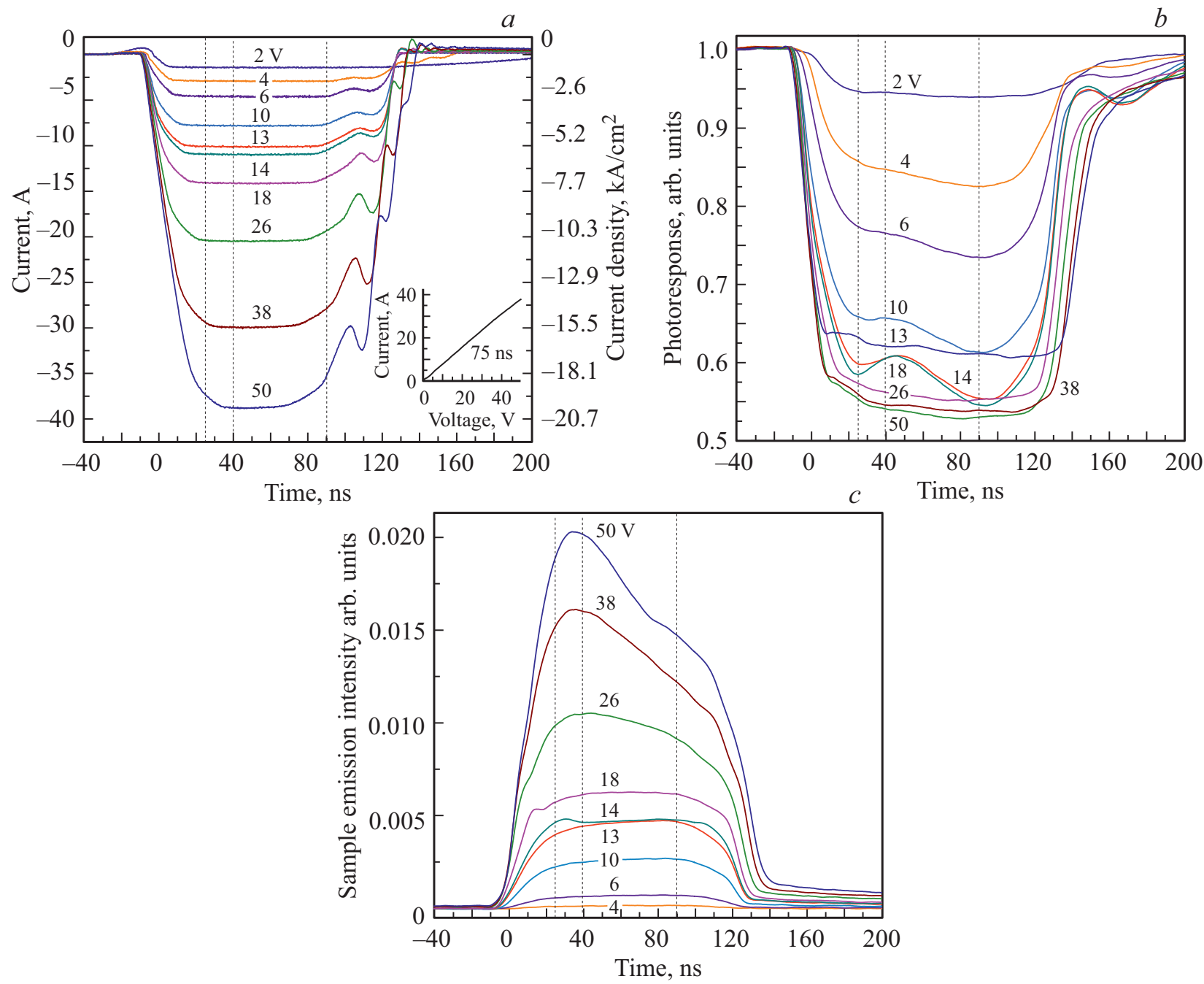

Рис. 3. Временнб́е зависимости тока $(a)$, фотоотклика от прошедшего через кристалл излучения $(b)$ и собственного излучения образца $(c)$ для случая работы образца с двумя подключенными сегментами.

сегментами позволяли накачивать каждый сегмент в отдельности либо несколько сегментов вместе. Ширина одиночного сегмента составляла 220 мкм, а его длина 440 мкм. Ширина пассивных областей также составляла

Составы и толщины слоев экспериментальной гетероструктуры

\begin{tabular}{l|c|c}
\hline \multicolumn{1}{c|}{ Назначение слоя } & Состав слоя & Толщина слоя \\
\hline$N$-эмиттер & $\mathrm{Al}_{0.4} \mathrm{Ga}_{0.6} \mathrm{As}$ & 1.5 мкм \\
$i$-волновод & $\mathrm{Al}_{0.3} \mathrm{Ga}_{0.7} \mathrm{As}$ & 1.9 мкм \\
$i$-спейсер & $\mathrm{GaAs}$ & $60 \AA$ \\
Квантовая яма & $\mathrm{InGaAs}$ & $90 \AA$ \\
$i$-барьерный слой & $\mathrm{GaAs}$ & $300 \AA$ \\
Квантовая яма & $\mathrm{InGaAs}$ & $90 \AA$ \\
$i$-спейсер & $\mathrm{GaAs}$ & $60 \AA$ \\
$i$-волновод & $\mathrm{Al}_{0.3} \mathrm{Ga}{ }_{0.7} \mathrm{As}$ & 1.2 мкм \\
$P$-эмиттер & $\mathrm{Al}_{0.55} \mathrm{Ga} 0.45 \mathrm{As}$ & 1 мкм \\
$P^{+}$-контактный слой & $\mathrm{GaAs}$ & 0.3 мкм
\end{tabular}

440 мкм. Таким образом, на пути прохождения оптического пучка оказывалось известное число прокачиваемых электрическим током сегментов от 1 до $\mathrm{N}$ с известными размерами области прокачки. На торцы кристаллов наносилось просветляющее покрытие AlN. Подготовленные кристаллы монтировались на медный теплоотвод с помощью индиевого припоя подложкой к теплоотводу.

\section{3. Ввод излучения с длиной волны 1560 нм}

\section{1. Модуляция излучения при вводе в центр полоска}

Предполагается, что концентрация носителей заряда при прокачке через образец электрического тока может быть обнаружена именно в области под полосковым контактом. На первом этапе зондирующее излучение вводилось в волноводный слой под сегментированным 


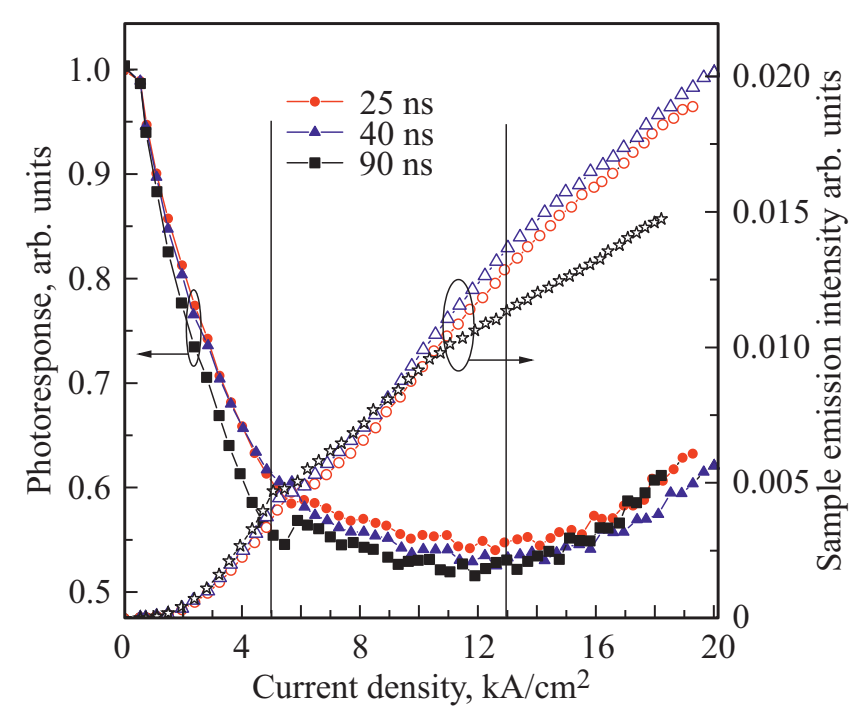

Рис. 4. Зависимость амплитуд фотоотклика от прошедшего через кристалл излучения (слева) и собственного излучения образца (справа) от плотности тока в моменты времени 25,40 и 90 нс для случая работы образца с двумя подключенными сегментами.

контактом и проводились измерения фотооткликов от прошедшего через кристалл зондирующего излучения для двух вариантов включения: 1) при одновременной накачке двух ближайших сегментов, что обеспечивало площадь области накачки $220 \times 880$ мкм $^{2}$ и плотность протекающего через образец тока от 0 до 20 кА/ $\mathrm{cm}^{2}$; 2) один из сегментов, используемых в варианте 1), отключался, и накачивался только один сегмент, что обеспечивало площадь области накачки $220 \times 440$ мкм $^{2}$ и плотность протекающего через образец тока от 0 до 40 кА/ $\mathrm{cm}^{2}$. В эксперименте выбиралось положение сегментов вблизи выходного зеркала образца, область за входной гранью оставалась непрокачиваемой. На рис. 3 можно видеть импульсы тока через накачиваемые сегменты (рис. 3,a), измеряемого на контрольном резисторе 1.25 Ом, фотоотклики на длине волны 1560 нм от прошедшего через кристалл зондирующего излучения (рис. $3, b)$ до (уровень 1.00) и во время модуляции поглощением на свободных носителях, а также динамику собственного излучения образца на длине волны 1010 нм (рис. 3,c) для нескольких плотностей тока при подключении двух сегментов. Для удобства сопоставления графиков используется такой параметр, как напряжение питания источника тока. Зависимость амплитуды тока источника от напряжения питания для момента времени 75 нс представлена на вставке к рис. $3, a$. На рис. $3, b$ за единицу принят уровень сигнала до начала модуляции.

Весь набор плотностей тока можно условно разделить на три диапазона: от 0 до $\sim 5$, от 5 до 13, от 13 до $20 \kappa \mathrm{A} / \mathrm{cm}^{2}$.

Диапазон плотностей тока от 0 до $\sim 5 \kappa \mathrm{A} / \mathrm{cm}^{2}$ характеризуется спадом амплитуды фотоотклика во время прохождения модулирующего импульса тока, близким к линейному (рис. 4). При плотности тока 2 кА/см² происходит достижение порога лазерной генерации. После преодоления порога лазерной генерации можно наблюдать возрастание амплитуды собственного излучения, форма импульса которого близка к форме импульса тока накачки, что может свидетельствовать о работе образца на модах резонатора Фабри-Перо (рис. 4).

В диапазоне плотностей тока от 5 до 13 кА/см ${ }^{2}$ к генерации на модах резонатора Фабри-Перо образца добавляется работа на модах иных конфигураций. В интервале 5-8 кА/см ${ }^{2}$ наблюдается отчетливо выраженный срыв генерации на модах Фабри-Перо (см. изменение формы импульса для диапазона $13-14$ В на рис. 3,c). Для образцов с сегментированным контактом и локальной накачкой характерно появление высокодобротных замкнутых мод [13]. Форма модуляции также существенно изменяется относительно формы импульсов тока: появляется заметное ослабление поглощения, максимум которого приходится на момент времени 45 нс. В этот момент также происходит спад амплитуды собственного излучения. Можно сделать вывод, что срыв генерации Фабри-Перо сопровождается снижением концентрации носителей заряда в области под полосковым контактом. Это согласуется с представлением о характерном поведении образца при возникновении замкнутых мод.

В диапазоне от 13 до $20 \mathrm{\kappa} / \mathrm{cm}^{2}$ мы видим заметное искажение формы оптического импульса собственного излучения. Первая половина импульса характеризуется работой преимущественно на модах резонатора ФабриПеро, эффективность которой снижается с течением времени. Поглощение на свободных носителях начинает ослабевать. Модулированный сигнал имеет слабовыраженные особенности на протяжении всей длительности импульса модулирующего тока. Причина этого явления в настоящий момент до конца не ясна. Однако можно высказать предположение, что, как и в случае с предыдущим диапазоном плотностей тока, мы наблюдаем работу образца как на модах Фабри-Перо, так и на замкнутых модах. Это приводит к постепенному уменьшению концентрации свободных носителей с увеличением плотности тока.

Отключение одного из двух сегментов привело к изменению динамики импульсов собственного излучения в сторону большего соответствия динамике тока, эффектов срыва генерации замечено не было. Кроме того, форма модуляции поглощением на свободных носителях не имела ярко выраженных особенностей во всем диапазоне плотностей тока. Вышеперечисленные факты свидетельствуют о более стабильном режиме работы образца с одним подключенным сегментом по сравнению со случаем работы на двух сегментах. Динамику тока, фотоотклика от прошедшего через образец излучения и собственного излучения образца можно видеть на рис. 5. Как и в предыдущем случае, были построены зависимости амплитуды фотоотклика и собственного излучения для моментов времени 25, 40 и 90 нс (рис. 6). Видно, что усиление поглощения на свободных носителях соответствует увеличению амплитуды собственного 

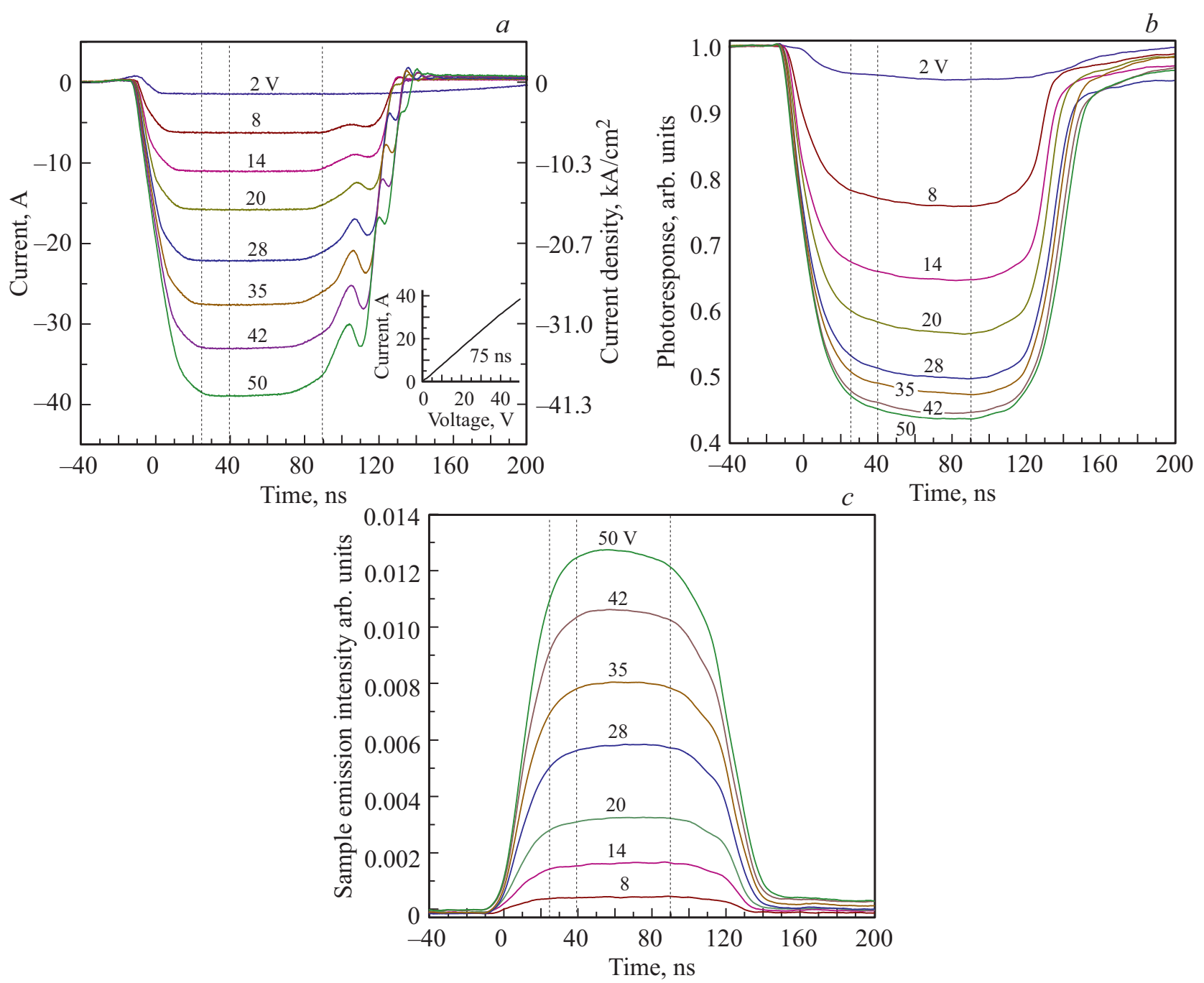

Рис. 5. Временнб́е зависимости тока $(a)$, фотоотклика от прошедшего через кристалл излучения $(b)$ и собственного излучения образца $(c)$ для случая работы образца с одним подключенным сегментом.

излучения, что характерно для работы лазера на модах Фабри-Перо, т. е. штатной работы лазера.

\section{2. Расчет поглощения в образце при протекании электрического тока}

После проведения измерений для случаев одного и двух подключенных сегментов была сделана оценка показателя поглощения по формуле (4). Согласно теории [11], показатель поглощения прямо пропорционален концентрации свободных носителей заряда:

$$
\alpha=\sigma_{n} n+\sigma_{p} p,
$$

где $n, p-$ концентрации свободных электронов и дырок, $\sigma_{n}, \sigma_{p}-$ сечения поглощения для электронов и дырок.

Тогда концентрация избыточных носителей заряда в активной области и волноводных слоях гетероструктуры определяется плотностью тока и должна увеличиваться при увеличении плотности тока, что дает пропорциональное увеличение показателя поглощения. Однако для случая полупроводниковых лазеров это справедливо при сохранении неизменными модовых структур. В противном случае изменение модовых конфигураций приведет к изменению пороговых условий и, как результат, к изменению пороговой концентрации в активной области, что может также повлиять на концентрацию в волноводных слоях. Из рис. 4 видно, что в случае двух подключенных сегментов при плотностях тока выше 5 кА/см ${ }^{2}$ образец существенно изменяет режим с ожидаемой и характерной для полупроводниковых лазеров работы на модах Фабри-Перо на работу в режимах с выраженными срывами генерации, поэтому для расчета показателя поглощения был выбран диапазон плотностей тока $0-2$ кА/ $\mathrm{cm}^{2}$, где наблюдалась наибольшая стабильность. Результаты расчета можно увидеть на рис. 7. Действительно, по мере возрастания плотности тока мы наблюдем линейный рост поглощения. Если продлить линию в точку 0 кА/см², то мы получим расчетный показатель поглощения при отсутствии протекающего тока. Это значение приблизительно можно оценить в $1.2 \mathrm{~cm}^{-1}$. 


\section{3. Ввод излучения в пассивную область}

Особенности работы образца при двух подключенных сегментах на высоких плотностях тока могут свидетельствовать о сложной пространственной динамике носителей заряда в образце. Мы провели дополнительный эксперимент, в котором излучение вводилось в непрокачиваемую током пассивную область, располагающуюся в стороне от сегментированного полоскового контакта, на расстоянии $>150$ мкм. Было замечено, что в пассивной области по мере нарастания плотности тока накачки полоскового контакта также появляются носители заряда, причем их динамика имеет сложную форму. На рис. 8 представлена зависимость амплитуды модулированного

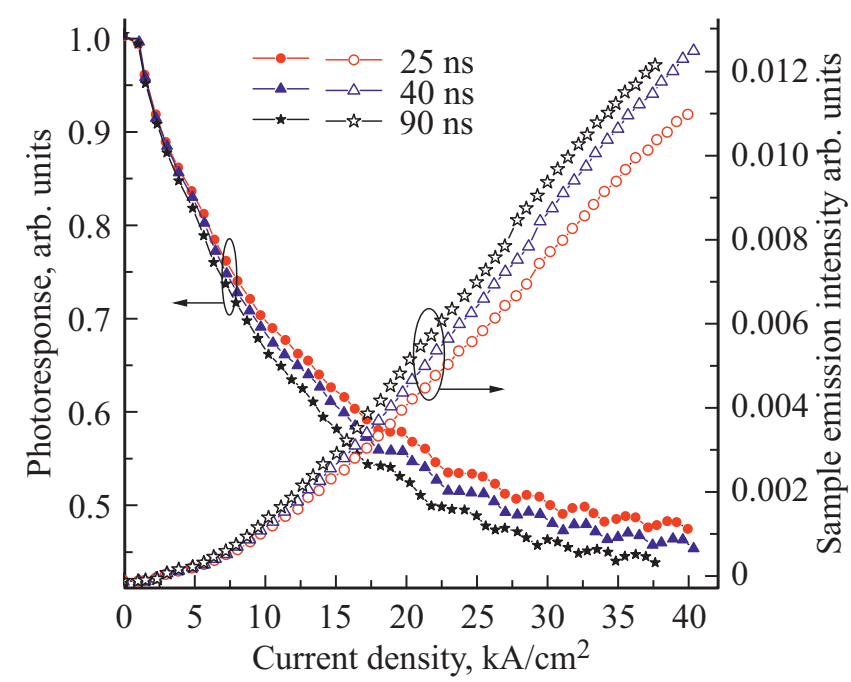

Рис. 6. Зависимость амплитуд фотоотклика от прошедшего через кристалл излучения (слева) и собственного излучения образца (справа) от плотности тока в моменты времени 25, 40 и 90 нс для случая работы образца с одним подключенным сегментом.

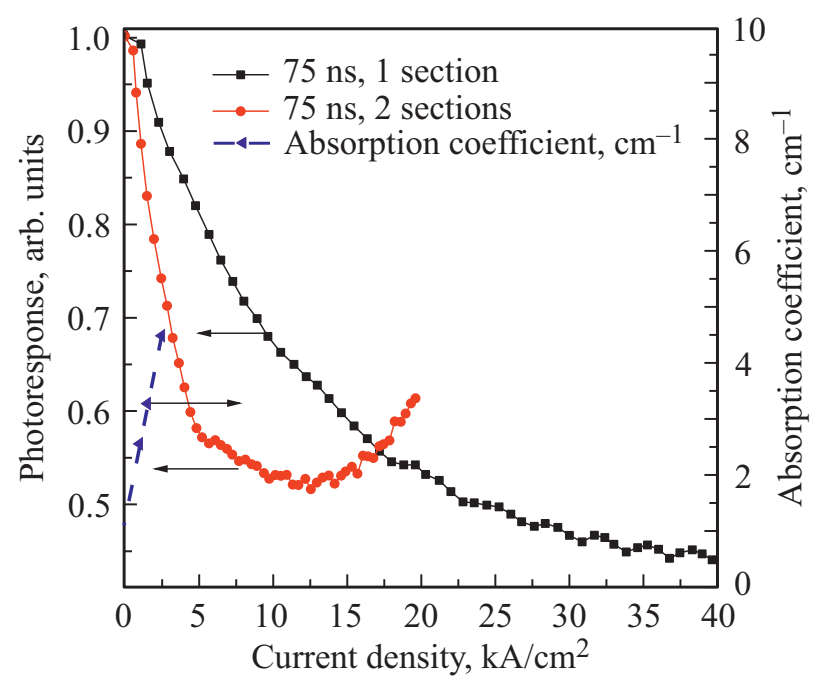

Рис. 7. Расчет показателя поглощения из сопоставления амплитуд модулированных фотооткликов для случаев подключения одного и двух сегментов.

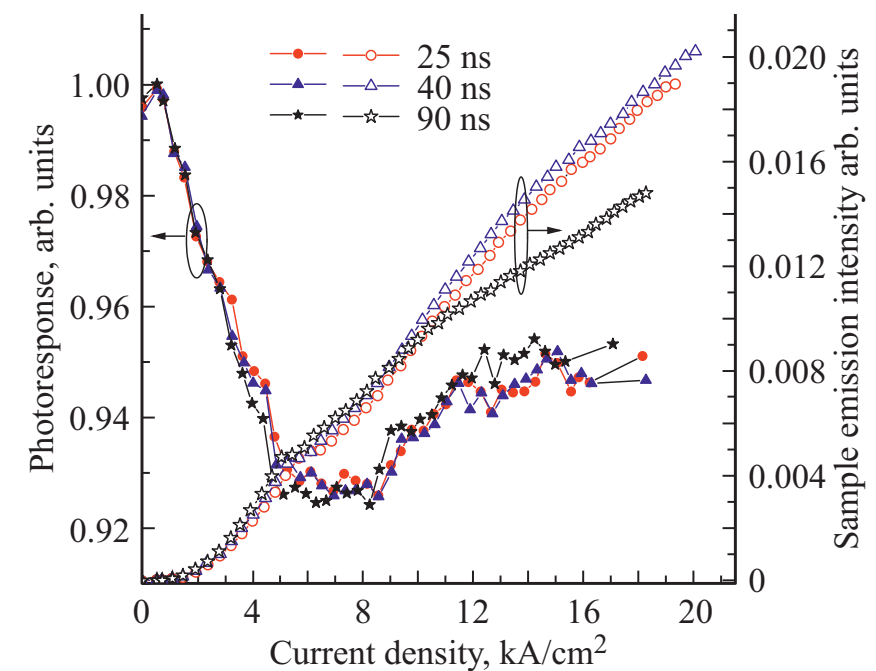

Рис. 8. Зависимость амплитуд фотоотклика от прошедшего через кристалл излучения (слева) и собственного излучения образца (справа) от плотности тока в моменты времени 25, 40 и 90 нс для случая работы образца с двумя подключенными сегментами и засветкой в пассивную область.

фотоотклика зондирующего излучения на длине волны 1560 нм для различных плотностей тока (слева). Для наглядности на этом же рисунке вновь приведены зависимости амплитуды собственного излучения на длине волны 1010 нм (справа) при накачке двух сегментов полоскового контакта. Указанные плотности тока, как и в предыдущих случаях, рассчитывались из отношения измеренных амплитуд тока к площади прокачиваемых сегментов. Однако наличие достаточно сильного поглощения на свободных носителях в пассивной области может свидетельствовать о более сложном распределении носителей заряда при высоких амплитудах тока. С одной стороны, это может быть связано с растеканием тока из области полоскового контакта, а с другой стороны, - c фотогенерацией носителей заряда за счет поглощения излучения из прокачиваемой части активной области или возникновения замкнутых мод на траектории зондирующего излучения. Примечательно, что поглощение на свободных носителях в пассивной области сначала нарастает с увеличением плотности тока, а затем уменьшается. Первоначально рост поглощения может быть связан с повышением интенсивности спонтанного излучения в активной области под полосковым контактом и соответственно усилением фотогенерации в активной области пассивной части. Рост поглощения замедляется вблизи точки срыва генерации на модах Фабри-Перо (плотность тока $>5 \mathrm{\kappa A} / \mathrm{cm}^{2}$ ), и при дальнейшем росте тока накачки наблюдается спад поглощения в пассивной части. Это может быть связано с включением замкнутых мод и, как следствие, снижением пороговой концентрации, что приводит к снижению потока спонтанного излучения в пассивную область и снижению скорости фотогенерации и концентрации носителей в активной области пассивной части структуры. 


\section{4. Заключение}

Ввод излучения в область непосредственно под сегментированным полосковым контактом показал существенное различие поведения образца в случаях одного и двух подключенных сегментов длиной 440 мкм и шириной 220 мкм. Если при одном подключенном сегменте во всем диапазоне плотностей тока наблюдалось нарастание концентрации свободных носителей заряда и амплитуды собственного излучения, то при двух подключенных сегментах происходили очевидные перестроения режимов работы. При двух подключенных сегментах в диапазоне плотностей тока от 0 до 5 кА/см ${ }^{2}$ мы видим штатный режим работы образца, сопровождающийся переходом от спонтанного излучения к лазерной генерации на модах резонатора ФабриПеро. При плотностях тока выше 5 кА/см ${ }^{2}$ происходят срывы генерации, вероятной причиной которых может быть появление отличных от Фабри-Перо модовых структур, в том числе и высокодобротных замкнутых мод. Поглощение на свободных носителях заряда в этом случае ослабевает за счет общего снижения уровня концентрации носителей в области засветки. Этот эффект наблюдается и в динамике модулированного фотоотклика от проходящего через образец излучения: при снижении интенсивности собственного излучения происходит уменьшение поглощения.

Ввод излучения в пассивную область на расстоянии 150 мкм от полоска продемонстрировал наличие поглощения на свободных носителях. Эксперименты показали сложный характер динамики носителей заряда пассивной области. До момента срыва лазерной генерации концентрация повышается, что, по-видимому, связано с эффективной фотогенерацией за счет спонтанного излучения из области под полосковым контактом. По мере ухудшения эффективности мод Фабри-Перо поглощение на свободных носителях ослабевает, что может быть связано со снижением пороговой концентрации под полосковым контактом и скорости фотогенерации в пассивной области при включении высокодобротных модовых структур.

Были проведены оценки показателя поглощения в зависимости от плотности тока для длины волны 1560 нм. Показано, что в диапазоне плотностей тока $0-2 \kappa \mathrm{A} / \mathrm{cm}^{2}$ поглощение меняется от 1.2 до $4.5 \mathrm{~cm}^{-1}$.

\section{Финансирование работы}

Работа частично выполнена при поддержке Российского фонда фундаментальных исследований (проект „мол_а“ № 18-38-00906 „Закономерности формирования и развития пространственных неоднородностей тока в низковольтных полупроводниковых гетероструктурах с нелинейной обратной связью“).

\section{Конфликт интересов}

Авторы заявляют об отсутствии конфликта интересов.

\section{Список литературы}

[1] Simon M. Sze, K. Ng. Kwok. Physics of semiconductor devices (John Wiley \& Sons, 2006).

[2] L.A. Coldren, S.W. Corzine, M.L. Mashanovitch. Diode lasers and photonic integrated circuits (John Wiley \& Sons, 2012).

[3] ATLAS User's Manual (Silvaco International, Sunnyvale, CA, 2015).

[4] J. Piprek, Z.M. Li. Phot. Tech. Lett., 30, 963 (2018).

[5] B. Ryvkin, E. Avrutin. Electron. Lett., 42, 1283 (2006).

[6] Д.А. Веселов, Н.А. Пихтин, А.В. Лютецкий, Д.Н. Николаев, С.О. Слипченко, 3.Н. Соколова, В.В. Шамахов, И.С. Шашкин, Н.В. Воронкова, И.С. Тарасов. Квант. электрон., 45 (7), 604 (2015)

[D.A. Veselov, N.A. Pikhtin, A.V. Lyutetskiy, D.N. Nikolaev, S.O. Slipchenko, Z.N. Sokolova, V.V. Shamakhov, I.S. Shashkin, N.V. Voronkova, I.S. Tarasov. Quant. Electron., 45 (7), 604 (2015).]

[7] D.A. Veselov, Yu.K. Bobretsova, A.Y. Leshko, V.V. Shamakhov, S.O. Slipchenko, N.A. Pikhtin. J. Appl. Phys., 126 (21), 213107 (2019).

[8] S.O. Slipchenko, A.A. Podoskin, O.S. Soboleva, N.A. Pikhtin, T.A. Bagaev, M.A. Ladugin, A.A. Marmalyuk, V.A. Simakov, I.S. Tarasov. J. Appl. Phys., 119 (12), 124513 (2016).

[9] S.O. Slipchenko, A.A. Podoskin, O.S. Soboleva, N.A. Pikhtin, T.A. Bagaev, M.A. Ladugin, A.A. Marmalyuk, V.A. Simakov, I.S. Tarasov. J. Appl. Phys., 121 (5), 054502 (2017).

[10] S. Vainshtein, V. Yuferev, J. Kostamovaara. IEEE Trans. Electron Dev., 50 (9), 1988 (2003).

[11] H. Wenzel, P. Crump, A. Pietrzak, C. Roder, X. Wang, G. Erbert. Optical Quant. Electron., 41 (9), 645 (2009).

[12] P.S. Gavrina, O.S. Soboleva, A.A. Podoskin, D.N. Romanovich, V.S. Golovin, S.O. Slipchenko, N.A. Pikhtin, T.A. Bagaev, M.A. Ladugin, A.A. Marmalyuk, V.A. Simakov. Tech. Phys. Lett., 45 (4), 374 (2019).

П.С. Гаврина, О.С. Соболева, А.А. Подоскин, Д.Н. Романович, В.С. Головин, С.О. Слипченко, Н.А. Пихтин, Т.А. Багаев, М.А. Ладугин, А.А. Мармалюк, В.А. Симаков. Письма ЖТФ, 45 (8), 7 (2019).

[13] А.А. Подоскин, Д.Н. Романович, И.С. Шашкин, П.С. Гаврина, 3.Н. Соколова, С.О. Слипченко, Н.А. Пихтин. ФТП, 53 (6), 828 (2019).

[A.A. Podoskin, D.N. Romanovich, I.S. Shashkin, P.S. Gavrina, Z.N. Sokolova, S.O. Slipchenko, N.A. Pikhtin. Semiconductors, 53 (6), 828 (2019).]

Редактор Л.В. Шаронова 


\section{Study of the spatial and current dynamics of optical losses in semiconductor laser heterostructures by optical probe}

P.S. Gavrina, O.S. Soboleva, A.A. Podoskin, A.E. Kazakova, V.A. Kapitonov, S.O. Slipchenko, N.A. Pikhtin

loffe Institute, 194021 St. Petersburg, Russia

Abstract The spatiotemporal dynamics of optical losses and carrier density in a heterostructure of a semiconductor laser with a segmented contact were studied using an optical probe technique based on the coupling of probe radiation with a wavelength of $1560 \mathrm{~nm}$ into a semiconductor laser chip under investigation based on an $\mathrm{AlGaAs} / \mathrm{InGaAs} / \mathrm{GaAs}$ heterostructure and emitting at wavelength of $1010 \mathrm{~nm}$. The use of a segmented design of the current pumping region made it possible to estimate the absolute value of internal optical losses. It was shown that a change in the configuration of the Fabry-Perot eigenmodes of the laser affects the distribution of charge carriers and internal optical losses both in the current pump region and in the passive part of the laser chip that is not pumped by the current. 